\title{
Detection of critical cerebral desaturation thresholds by three regional oximeters during hypoxia: a pilot study in healthy volunteers
}

Kerry L. Tomlin, Anna-Maria Neitenbach and UIf Borg ${ }^{*}$ (D)

\begin{abstract}
Background: Regional oximetry is increasingly used to monitor post-extraction oxygen status of the brain during surgical procedures where hemodynamic fluctuations are expected. Particularly in cardiac surgery, clinicians employ an interventional algorithm to restore baseline regional oxygen saturation $\left(\mathrm{rSO}_{2}\right)$ when a patient reaches a critical desaturation threshold. Evidence suggests that monitoring cardiac surgery patients and intervening to maintain $\mathrm{rSO}_{2}$ can improve postoperative outcomes; however, evidence generated with one manufacturer's device may not be applicable to others. We hypothesized that regional oximeters from different manufacturers respond uniquely to changes in oxygen saturation in healthy volunteers.

Methods: Three devices were tested: INVOS ${ }^{\mathrm{TM}} 5100 \mathrm{C}$ (Medtronic), EQUANOX 7600 (Nonin), and FORE-SIGHT ${ }^{\mathrm{TM}}$ (CASMED) monitors. We divided ten healthy subjects into two cohorts wearing a single sensor each from INVOS and EQUANOX $(n=6)$, or INVOS and FORE-SIGHT $(n=4)$. We induced and reversed hypoxia by adjusting the fraction of inspired oxygen. We calculated the magnitude of absolute $\mathrm{rSO}_{2}$ change and rate $\mathrm{of}_{\mathrm{rSO}}$ change during desaturation and resaturation, and determined if and when each device reached a critical interventional $\mathrm{rSO}_{2}$ threshold during hypoxia.
\end{abstract}

Results: All devices responded to changes in oxygen directionally as expected. The median absolute $\mathrm{rSO}_{2}$ change and the rate of $\mathrm{rSO}_{2}$ change was significantly greater during desaturation and resaturation for INVOS compared with EQUANOX $(P=0.04)$. A similar but nonsignificant trend was observed for INVOS compared with FORE-SIGHT; our study was underpowered to definitively conclude there was no difference. A $10 \%$ relative decrease in $\mathrm{rSO}_{2}$ during desaturation was detected by all three devices across the ten subjects. INVOS met a 20\% relative decrease threshold in all subjects of both cohorts, compared to 1 with EQUANOX and 2 with FORE-SIGHT. Neither EQUANOX nor FORE-SIGHT reached a 50\% absolute $\mathrm{rSO}_{2}$ threshold compared with 4 and 3 subjects in each cohort with INVOS, respectively.

Conclusions: Significant differences exist between the devices in how they respond to changes in oxygen saturation in healthy volunteers. We suggest caution when applying evidence generated with one manufacturer's device to all devices.

Keywords: Regional oximetry, Cerebral oximetry, Near infrared spectroscopy, Cerebral desaturation, Hypoxia

\footnotetext{
* Correspondence: ulf.borg@medtronic.com

Department of Medical Affairs, Patient Monitoring \& Recovery, Medtronic,

6135 Gunbarrel Ave, Boulder, CO 80301, USA
}

(c) The Author(s). 2017 Open Access This article is distributed under the terms of the Creative Commons Attribution 4.0 International License (http://creativecommons.org/licenses/by/4.0/), which permits unrestricted use, distribution, and reproduction in any medium, provided you give appropriate credit to the original author(s) and the source, provide a link to the Creative Commons license, and indicate if changes were made. The Creative Commons Public Domain Dedication waiver (http://creativecommons.org/publicdomain/zero/1.0/) applies to the data made available in this article, unless otherwise stated. 


\section{Background}

A recent publication reported that surveyed cardiac anesthesiologists and perfusionists view regional oximetry as useful or essential for non-invasive monitoring of cerebral oxygen status during surgery [1]. Rapidly changing hemodynamic conditions can cause cerebral desaturation during cardiac, arthroscopic shoulder, major abdominal, and total knee replacement surgeries [2-5]. Originally published in 2007, clinicians continue to refine an interventional algorithm for maintaining cerebral oxygen saturation values $\left(\mathrm{rSO}_{2}\right)$ close to baseline during cardiac surgery by manipulating oxygen supply and demand [6, 7]. Cerebral desaturation-often defined as a $20 \%$ relative decrease from baseline or an absolute $\mathrm{rSO}_{2}$ value of $50 \%$ - may trigger clinicians to consider a range of possible interventions to restore oxygen saturation, such as ruling out mechanical obstructions or increasing mean arterial pressure [7]. Several cardiac studies suggest postoperative outcomes may improve when cerebral oxygen saturation is monitored and desaturation episodes are recognized and reversed, compared to no or blinded monitoring [8-13].

We propose that published evidence generated with one manufacturer's regional oximeter is unique to that device since they may reach an interventional threshold at different times or not at all. Each manufacturer uses a proprietary algorithm, as well as different emitter/detector spacing, number of wavelengths, and light source. We hypothesize that regional oximeters will react differently to clinically challenging situations where $\mathrm{rSO}_{2}$ is fluctuating.

To test our hypothesis, we subjected healthy volunteers to two cycles of desaturation and resaturation to characterize the differences in absolute $\mathrm{rSO}_{2}$ change and rate of $\mathrm{rSO}_{2}$ change between three $\mathrm{FDA}$-cleared regional oximeters: INVOS ${ }^{\mathrm{Tm}}$ 5100C (Medtronic), EQUANOX ${ }^{\mathrm{mx}}$ 7600 (Nonin), and FORE-SIGHT ${ }^{\mathrm{mm}}$ (CASMED) monitors. Changes in $\mathrm{rSO}_{2}$ were compared with changes in oxygen saturation as measured by pulse oximetry. Subjects wore one sensor each from two devices. We also took a unique approach from other comparative studies by tracking the time in which the devices reached critical $\mathrm{rSO}_{2}$ thresholds and whether the thresholds were met by one or both devices in individual subjects.

\section{Methods}

The Western Institutional Review Board (Puyallup, WA) approved an overarching protocol for a study of pulse oximetry devices, of which this was studied under. Written informed consent was obtained prior to participation. Twelve healthy subjects were enrolled from an existing hypoxia research pool and studied at the Medtronic Respiratory \& Monitoring Solutions clinical laboratory (Boulder, CO) from April 16 to 18, 2013. Two subjects were withdrawn: one failed screening, and the other experienced tachycardia and anxiety during the study. The study pool included males and non-pregnant or -lactating females, 18 to 50 years of age, from various racial and ethnic backgrounds, who tolerated hypoxia in previous studies. Skin pigmentation was rated as very light, olive, dark olive, or extremely dark.

Subjects reclined to approximately $20^{\circ}$ in the supine position with legs elevated. Peripheral capillary oxygen saturation $\left(\mathrm{SpO}_{2}\right)$ was monitored using the Nellcor N600 $\mathrm{x}^{\mathrm{Tm}}$ and Max- $\mathrm{A}^{\mathrm{max}}$ sensors (Medtronic, Dublin, Ireland) on the middle and index fingers. Standard monitoring included continuous electrocardiography, noninvasive blood pressure, end-tidal carbon dioxide, and respiration rate.

We tested three FDA-cleared regional oximeters: INVOS 5100C (Medtronic, Dublin, Ireland), EQUANOX 7600 (Nonin Medical, Plymouth, MN), and FORE-SIGHT (CASMED, Branford, CT). All devices report regional oxygen saturation $\left(\mathrm{rSO}_{2}\right)$ of the tissue beneath the sensor based on near infrared spectroscopy, the technical aspects of which are reported elsewhere [14, 15]. Two monitors were tested per subject with one INVOS sensor (SAFB$\mathrm{SM}$ ) on the left side of the forehead, and either an EQUANOX (8000CA or 8004CA) or FORE-SIGHT (standard large, medium, or small) sensor on the right, placed $2 \mathrm{~cm}$ apart if possible (Fig. 1). Six were studied with INVOS and EQUANOX, and four with INVOS and FORE-SIGHT.

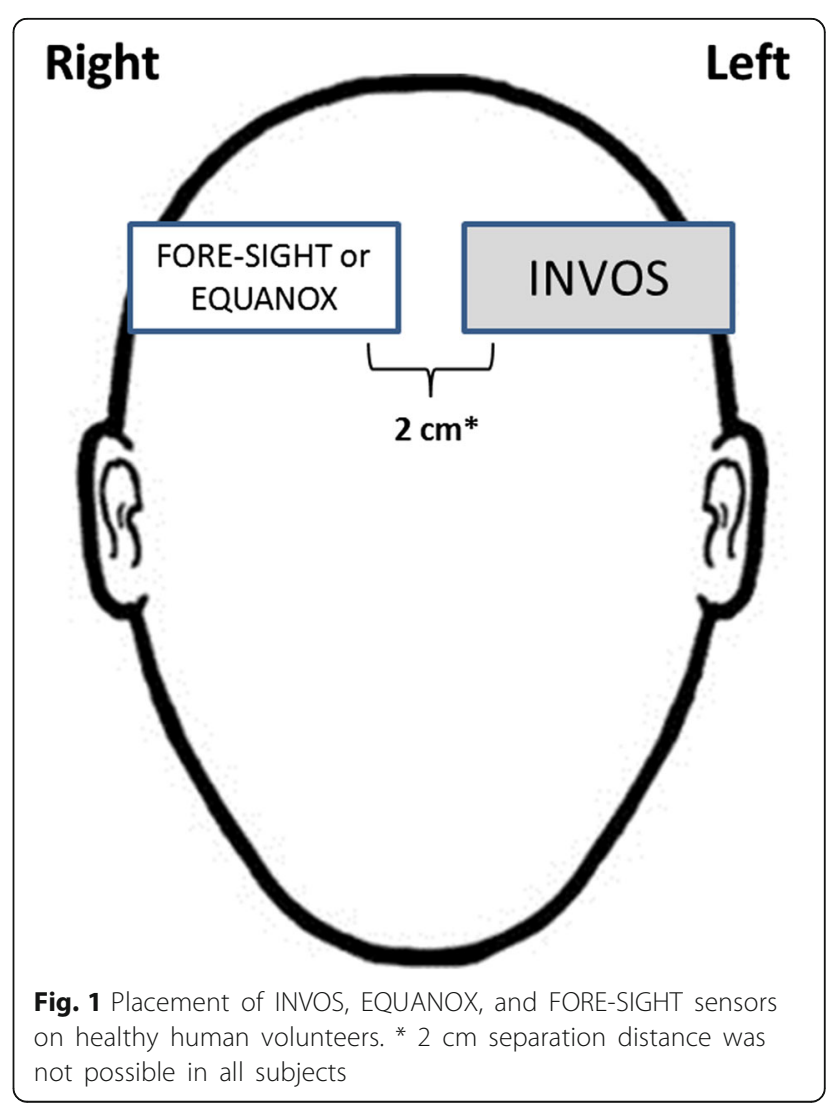


Subjects were fitted with a gas delivery mask, through which the anesthesiologist adjusted the fraction of inspired oxygen $\left(\mathrm{FiO}_{2}\right)$ using an oxygen-nitrogen gas mixer. The hypoxia protocol used meets the guideline defined in the ISO standard 80601-2-61:2011 for noninvasive laboratory testing on healthy volunteers for pulse oximetry, and is outlined in Fig. 2. (1) The initial baseline readings were established while breathing room air through the mask. (2) The $\mathrm{FiO}_{2}$ was decreased, based on the subject's tolerance, to achieve an $\mathrm{SpO}_{2}$ reading of approximately 70\%. (3) $\mathrm{SpO}_{2}$ was maintained at 70\% for at least $60 \mathrm{~s}$ after the reading plateaued, up to $5 \mathrm{~min}$. (4) The $\mathrm{FiO}_{2}$ was instantly increased to 1.0 to achieve a fraction of exhaled oxygen concentration $\left(\mathrm{FeO}_{2}\right)$ of at least 0.85. (5-9) Plateau measurements were collected, and the desaturation cycle was repeated. (10) The mask was removed, and data collection continued for $5 \mathrm{~min}$ with the subject breathing room air. The anesthesiologist and lab clinician monitored vital signs and subject comfort throughout the experiment. Digital trending data from all monitors were collected and time-stamped.

Statistical analyses were performed using Minitab 17 (Minitab, Inc, Sate College, PA). Baseline $\mathrm{rSO}_{2}$ and absolute percent changes in $\mathrm{SpO}_{2}$ and $\mathrm{rSO}_{2}$ were graphed as individual subject data and the median for each device within the cohort. Rates of absolute oxygen saturation change (\% per minute) were reported as the median and 95\% confidence interval (CI) for each device within the cohort. Given the small sample size, normality was not assumed and the Wilcoxon Signed-Rank test for paired samples was used to assess significant differences in baseline $\mathrm{rSO}_{2}$ values, absolute $\mathrm{rSO}_{2}$ changes, and rates of $\mathrm{rSO}_{2}$ change between the devices within each cohort. Significant differences between devices in detecting critical $\mathrm{rSO}_{2}$ thresholds were assessed using the exact binomial test. A $P$ value of $<0.05$ was considered statistically significant for all analyses.

\section{Results}

We performed two rounds of desaturation and resaturation in ten healthy human subjects. The INVOS/EQUANOX and INVOS/FORE-SIGHT cohorts were similar in subject characteristics and gender distributions (Table 1). We included Caucasian (70\%) and Asian (30\%) subjects with a range of skin pigmentation from very light to dark olive; no African Americans were available to participate.

\section{Baseline $\mathrm{rSO}_{2}$ prior to desaturation}

We plotted individual baseline $\mathrm{rSO}_{2}$ and median $\mathrm{rSO}_{2}$ values to visually compare the range and central tendency for each device (Fig. 3). The median baseline $\mathrm{rSO}_{2}$ prior to desaturation trended higher for FORE-SIGHT by approximately 10 percentage points compared with INVOS, although the differences were not statistically significant. Median INVOS and EQUANOX baseline $\mathrm{rSO}_{2}$ values differed by less than 2 percentage points. INVOS reported a wider range of individual baseline $\mathrm{rSO}_{2}$ values (54-93\%) compared to EQUANOX (58-84\%). FORE-SIGHT reported a narrower range of baseline values (69-81\%) than both INVOS and EQUANOX.

\section{Absolute \% change in $\mathrm{rSO}_{2}$ during desaturation and resaturation}

INVOS reported a significantly greater median absolute percent $\mathrm{rSO}_{2}$ change during both desaturation cycles compared with EQUANOX $(P=0.04)$, with a trend towards a greater change compared with FORE-SIGHT $(P=0.10)$ (Fig. 4). Although we did not compare the values statistically, the absolute percent change reported by INVOS (19.5-22.5\%) followed closer in magnitude to the absolute

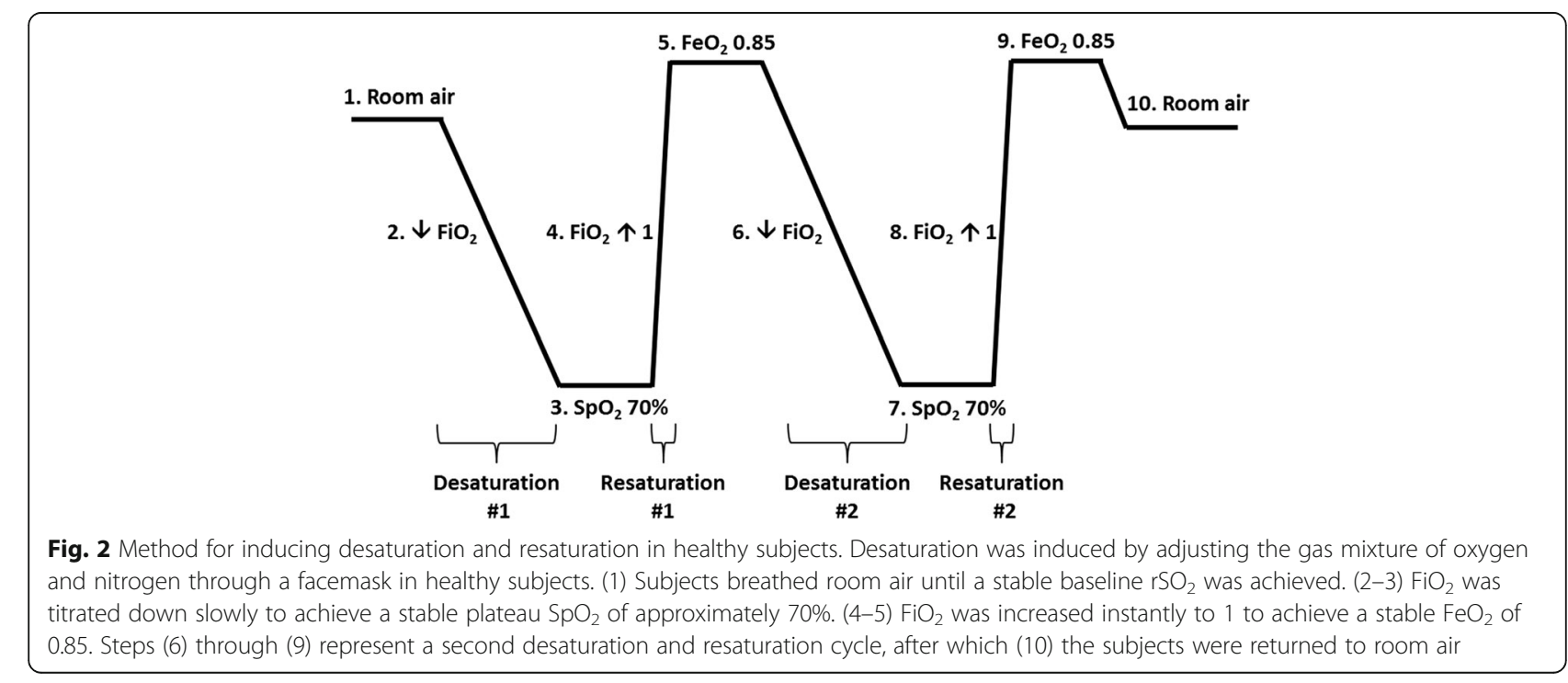


Table 1 Descriptive characteristics of study participants, mean \pm SD or $n(\%) ; N=10$

\begin{tabular}{lll}
\hline Characteristic & $\begin{array}{l}\text { INVOS/EQUANOX } \\
(n=6)\end{array}$ & $\begin{array}{l}\text { INVOS/FORESIGHT } \\
(n=4)\end{array}$ \\
\hline Age (yrs) & $35.2 \pm 10.2$ & $31.8 \pm 7.9$ \\
Male & $3(50 \%)$ & $2(50 \%)$ \\
Race/ethnicity & & $1(25 \%)$ \\
$\quad$ Asian & $2(33 \%)$ & $3(75 \%)$ \\
$\quad$ Caucasian & $4(67 \%)$ & \\
Skin pigmentation & & $3(75 \%)$ \\
$\quad$ Very light & $4(68 \%)$ & $0(0 \%)$ \\
$\quad$ Olive & $1(16 \%)$ & $1(25 \%)$ \\
$\quad$ Dark olive & $1(16 \%)$ & $176.6 \pm 11.5$ \\
Height (cm) & $172.6 \pm 4.7$ & $68.3 \pm 7.1$ \\
Weight $(\mathrm{kg})^{a}$ & $75.1 \pm 20.6$ & \\
\hline
\end{tabular}

${ }^{a}$ Weight was unavailable for one participant

changes in $\mathrm{SpO}_{2}$ (21.0-24.9\%) compared with EQUANOX (12.0-15.5\%) and FORE-SIGHT (13.5-15.0\%). We discovered similar results during the two resaturation cycles, although the difference in absolute percent change in $\mathrm{rSO}_{2}$ for INVOS compared with EQUANOX during resaturation \#2 only trended towards significance $(P=0.06)($ Fig. 5).

Rate of $\mathrm{rSO}_{2}$ change during desaturation and resaturation We report the rates of $\mathrm{rSO}_{2}$ change during desaturation for each of the devices in Table 2, with $\mathrm{SpO}_{2}$ change shown for comparison. For both desaturation cycles, INVOS reported a significantly greater percent $\mathrm{rSO}_{2}$ change than EQUANOX $(P=0.04)$, with a trend towards a greater change than FORE-SIGHT $(P=0.10)$. The median rates of $\mathrm{rSO}_{2}$ change for INVOS $(3.0-4.0 \% / \mathrm{min})$ followed closer in magnitude to rates of $\mathrm{SpO}_{2}$ change $(3.6-4.3 \% / \mathrm{min})$ than EQUANOX $(2.3-3.0 \% / \mathrm{min})$ or FORE-SIGHT $(2.2-2.4 \% / \mathrm{min})$.

The rate of $\mathrm{rSO}_{2}$ change during resaturation is reported in Table 3. INVOS reported a significantly greater rate of change compared with EQUANOX during resaturation $\# 1(P=0.04)$, with a trend towards a greater rate during resaturation $\# 2(P=0.06)$. Although numerically higher than FORE-SIGHT, the rates of $\mathrm{rSO}_{2}$ change for INVOS during both resaturation cycles were not statistically different. The median rates of $\mathrm{rSO}_{2}$ change for INVOS $(9.6-15.9 \% / \mathrm{min})$ were closer in magnitude to rates of $\mathrm{SpO}_{2}$ change $(11.75-19.1 \% / \mathrm{min})$ than EQUANOX $(9.2-11.2 \% / \mathrm{min})$ or FORE-SIGHT $(6.6-9.5 \% / \mathrm{min})$.

\section{Critical desaturation thresholds}

We reviewed the $\mathrm{rSO}_{2}$ values from desaturation \#1 for the following thresholds: 1) $10 \%$ relative change from baseline, 2) $20 \%$ relative change from baseline, and 3) an absolute value of $50 \%$. For each subject, we determined whether the devices reached the threshold, and the mean time difference when the threshold was met by both devices.

We considered a $10 \%$ relative change from baseline as an early indicator that cerebral oxygen saturation is decreasing and may require early intervention [2]. All three devices reached the $10 \%$ relative change in all subjects of both cohorts. On average, INVOS reached the $10 \%$ threshold $28 \mathrm{~s}$ earlier than EQUANOX, and $43 \mathrm{~s}$ earlier than FORE-SIGHT.
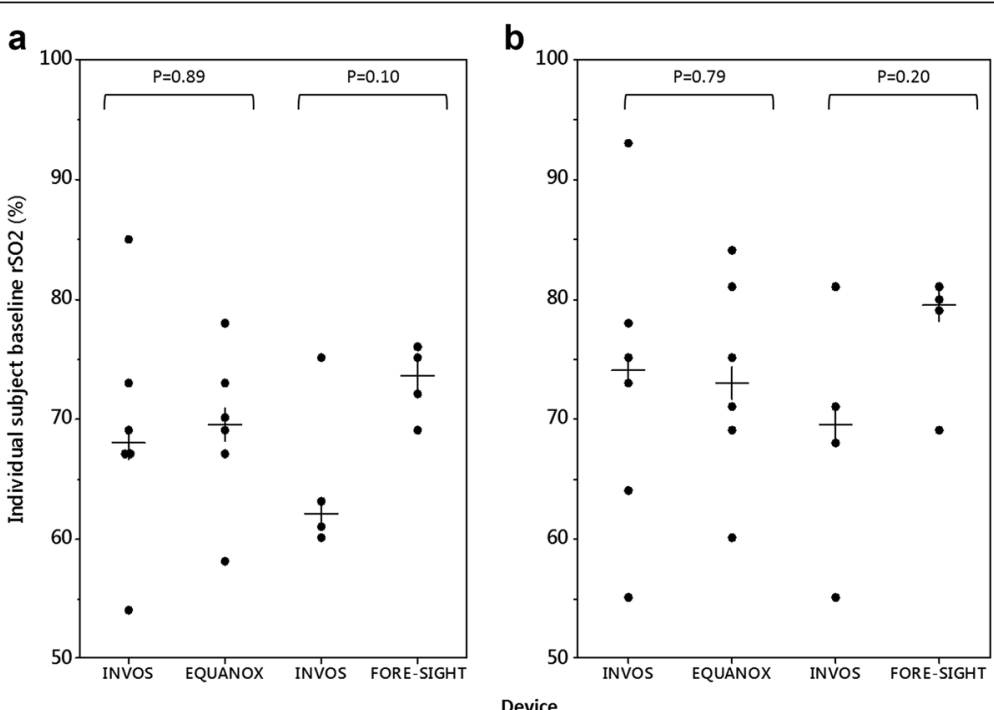

Fig. 3 Individual baseline and median $\mathrm{rSO}_{2}$ values prior to desaturation \#1 (a) and \#2 (b). INVOS demonstrated a wider spread of individual baseline values than both EQUANOX and FORE-SIGHT. Although the median FORE-SIGHT baseline $\mathrm{rSO}_{2}$ was consistently higher than INVOS by approximately 10\%, the differences were not statistically significant (two-tailed Wilcoxon Signed-Rank test for paired samples) 


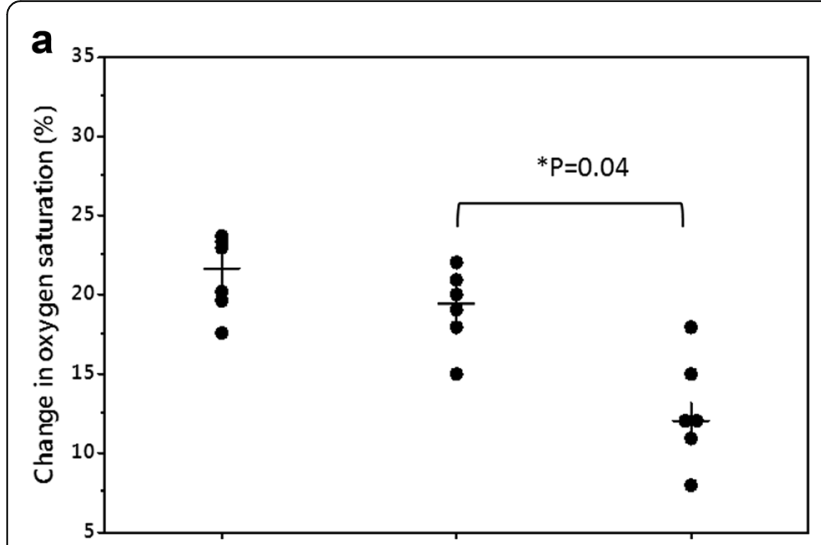

b
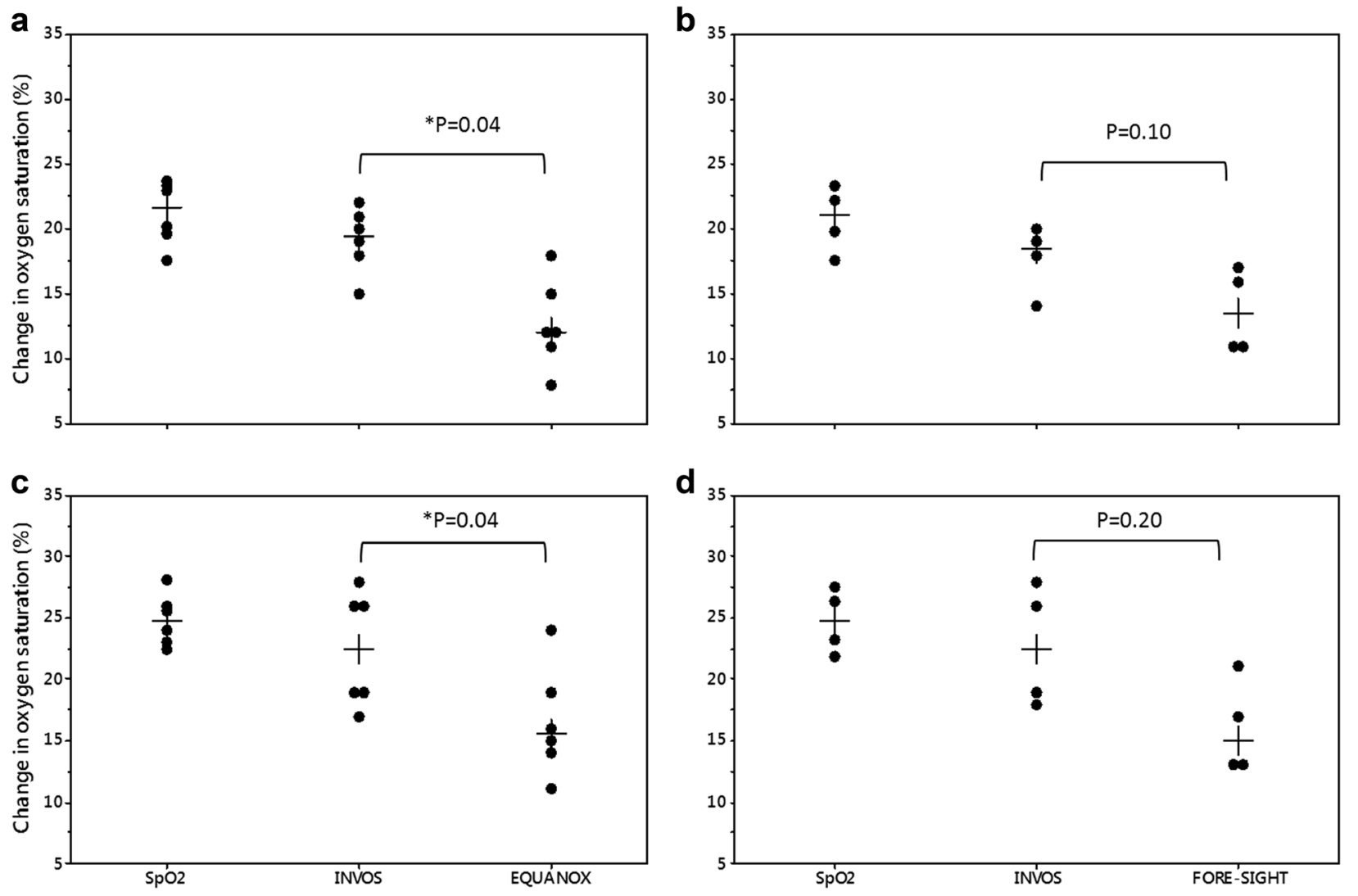

d

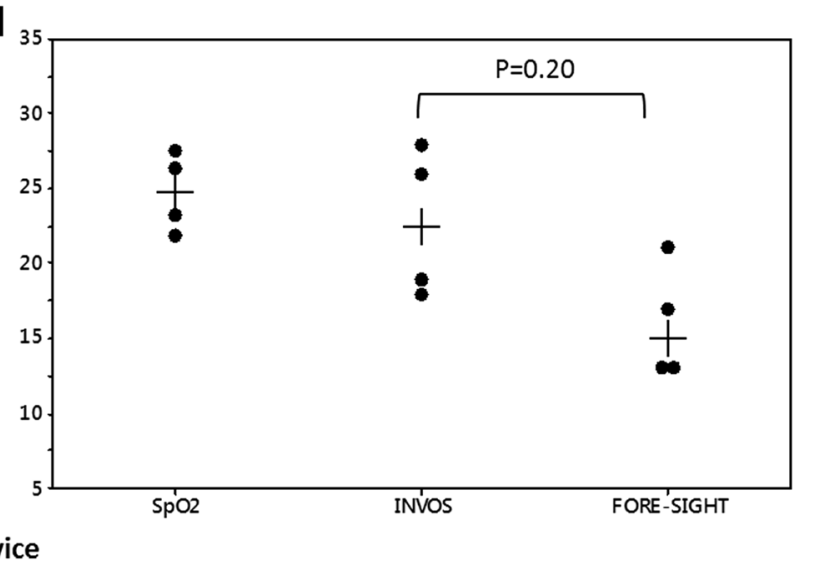

Fig. 4 Individual absolute and median $\mathrm{rSO}_{2}$ changes during desaturation \#1 (a, b) and \#2 (c, d). Absolute changes in SpO $\mathrm{S}_{2}$, representing global saturation, are shown for comparison. Median absolute change in $\mathrm{rSO}_{2}$ reported by INVOS during desaturation \#1 and \#2 were significantly greater than that of EQUANOX. Although FORE-SIGHT reported a smaller median absolute change during desaturation \#1 and \#2 compared with INVOS, the differences were not statistically significant (two-tailed Wilcoxon Signed-Rank test for paired samples). * Indicates statistical significance

Table 4 shows the number of subjects in which only one monitor for each pair detected a critical drop in $\mathrm{rSO}_{2}$. In the INVOS/EQUANOX cohort, INVOS met the $20 \%$ relative decrease for all six subjects in the cohort. In five of six subjects, EQUANOX did not detect a $20 \%$ relative decrease. There were no subjects for whom EQUANOX reached the threshold and INVOS did not, and although numerically different, the results only trended towards statistical significance $(P=0.06)$. We found similar results with the INVOS/FORE-SIGHT cohort, where INVOS met the $20 \%$ threshold in all four subjects compared with two subjects with FORE-SIGHT. There were no subjects for whom FORE-SIGHT detected a $20 \%$ relative change when INVOS did not. The difference was not statistically significant.

When we used an absolute threshold of $50 \%$, INVOS reached the threshold in four of six subjects, whereas EQUANOX did not in any subjects, with a trend towards statistical significance $(P=0.06)$. In the INVOS/FORESIGHT cohort, INVOS reached the threshold in three of four subjects, while FORE-SIGHT did not in any subjects. Again, the results were not statistically significant.

\section{Discussion}

All three devices reported changes in $\mathrm{rSO}_{2}$ during the course of desaturation and resaturation. We found that the median absolute change in $\mathrm{rSO}_{2}$, and the rate of $\mathrm{rSO}_{2}$ change per minute, was significantly greater with INVOS compared with EQUANOX during desaturation. The magnitude and rate of change was numerically greater with INVOS compared to FORE-SIGHT, but the differences were not statistically significant. When we reversed desaturation, we found similar significant differences in the magnitude and rate of change between INVOS and EQUANOX, with a trend towards significance between INVOS and FORE-SIGHT.

The most interesting finding was the discordance in detecting critical desaturation thresholds during desaturation when comparing devices on the same subject. All three technologies detected a minimum $10 \%$ relative decrease in $\mathrm{rSO}_{2}$ from baseline in all subjects; EQUANOX and FORE-SIGHT reached the threshold on average 28 to $43 \mathrm{~s}$ after INVOS, respectively. INVOS detected a $20 \%$ relative decrease in $\mathrm{rSO}_{2}$ in all subjects 

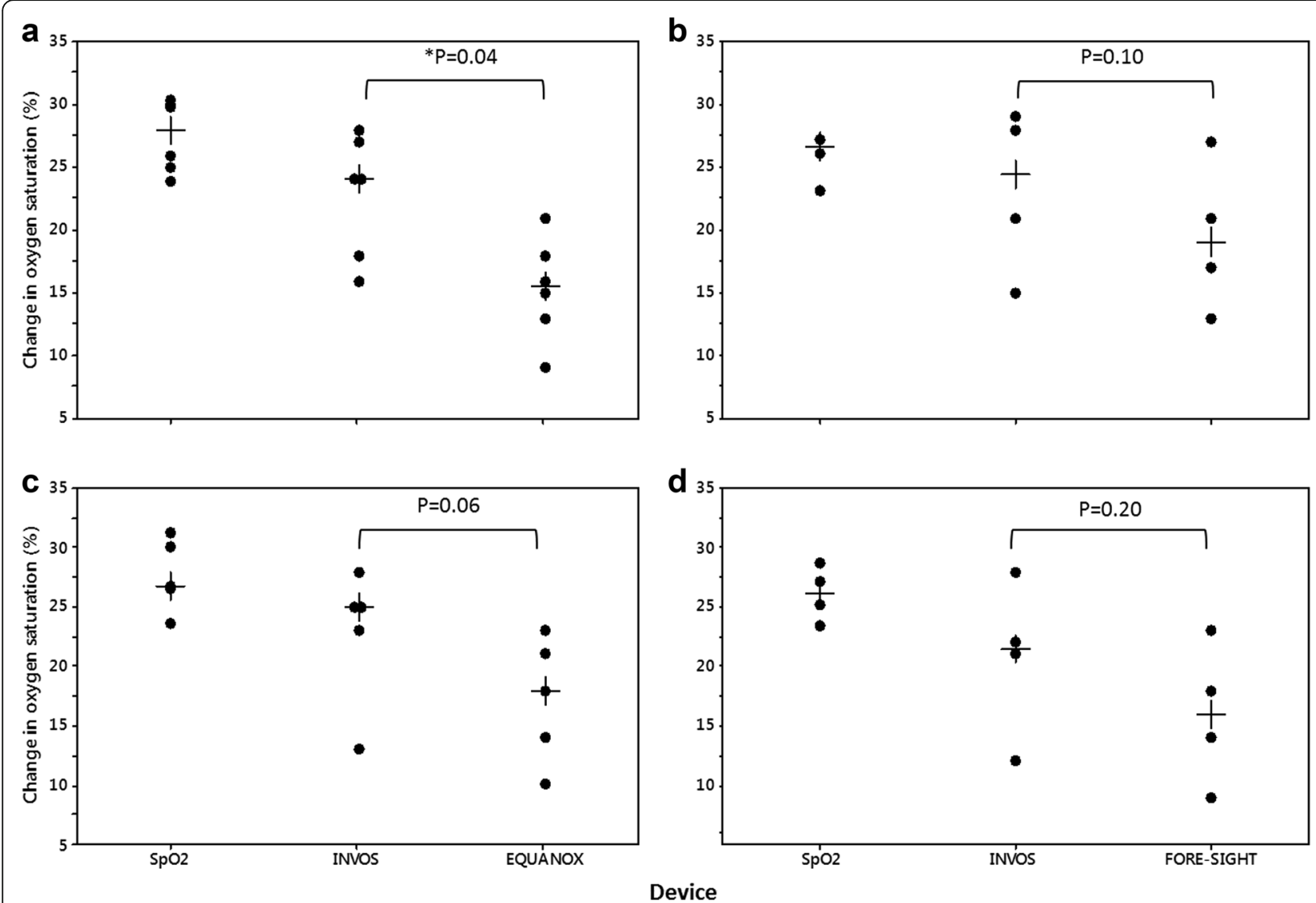

Fig. 5 Individual absolute and median $\mathrm{rSO}_{2}$ changes during resaturation \#1 (a, b) and \#2 (c, d). Absolute changes in $\mathrm{SpO}_{2}$, representing global saturation, are shown for comparison. Median absolute change in $\mathrm{rSO}_{2}$ reported by INVOS during resaturation \#1 was significantly greater than that of EQUANOX. Although FORE-SIGHT reported a smaller median absolute change during resaturation \#1 and \#2 compared with INVOS, the differences were not statistically significant (two-tailed Wilcoxon Signed-Rank test for paired samples). Data are missing for one INVOX/EQUANOX subject in graph C. *Indicates statistical significance

Table 2 Median rates ( $95 \% \mathrm{Cl}$ ) of absolute oxygen saturation change during desaturation

\begin{tabular}{lllll}
\hline & $\mathrm{SpO}_{2}$ & INVOS & EQUANOX & \\
$\begin{array}{l}\text { INVOS/EQUANOX } \\
(n=6)\end{array}$ & $\% / \mathrm{min}$ & $\% / \mathrm{min}$ & $\% / \mathrm{min}$ & $P$ value \\
Desaturation \#1 & $4.2(2.7,5.2)$ & $3.5(2.9,4.3)$ & $2.3(1.7,2.8)$ & $0.04^{\mathrm{a}}$ \\
Desaturation \#2 & $4.3(3.5,5.5)$ & $4.0(3.0,4.7)$ & $3.0(2.0,3.5)$ & $0.04^{\mathrm{a}}$ \\
& $\mathrm{SpO}_{2}$ & INVOS & FORE-SIGHT & \\
& $\% / \mathrm{min}$ & $\% / \mathrm{min}$ & $\% / \mathrm{min}$ & $P$ value \\
$\begin{array}{l}\text { INVOS/FORE-SIGHT } \\
(n=4)\end{array}$ & & & & \\
Desaturation \#1 & $3.6(2.9,4.1)$ & $3.0(2.5,3.5)$ & $2.4(1.8,2.8)$ & 0.10 \\
Desaturation \#2 & $3.7(2.9,5.0)$ & $3.4(2.4,4.9)$ & $2.2(1.7,4.0)$ & 0.10 \\
\hline
\end{tabular}

andicates statistical significance between devices (two-tailed Wilcoxon SignedRank test for paired samples) of both cohorts, compared to one with EQUANOX, and two subjects with FORE-SIGHT. Neither EQUANOX nor FORE-SIGHT met the $50 \%$ absolute $\mathrm{rSO}_{2}$ threshold in any subjects, compared to four and three subjects with INVOS, respectively. While our findings lacked statistical significance, they may have important clinical implications.

The randomized, controlled evidence reporting improved postoperative outcomes compared with no monitoring is based on detecting a critical drop in $\mathrm{rSO}_{2}$ in the monitored subjects that the clinician observes and intervenes to reverse $[4,8-12]$. These studies usually cite a threshold of either a 20 to $25 \%$ relative decrease from baseline, or absolute value of 50 to $60 \%$. Our study has shown that regardless of whether a relative or absolute threshold is used, disparities may exist between devices in detecting desaturation events. These disparities may result in clinicians intervening, for example, earlier and/ or more often when using one device compared to another. A clinician may employ cerebral oximetry to 
Table 3 Median rates ( $95 \% \mathrm{Cl}$ ) of absolute oxygen saturation change during resaturation

\begin{tabular}{|c|c|c|c|c|}
\hline & $\mathrm{SpO}_{2}$ & INVOS & EQUANOX & \\
\hline INVOS/EQUANOX $(n=6)$ & $(\% / \min )$ & $(\% /$ min $)$ & $(\% /$ min) & $P$ value \\
\hline Resaturation \#1 & $19.1(13.8,25.7)$ & $15.9(12.5,18.5)$ & $11.2(7.0,13.4)$ & $0.04^{\mathrm{a}}$ \\
\hline \multirow[t]{2}{*}{ Resaturation \#2 } & $15.3(11.6,21.9)$ & $12.7(8.5,17.7)$ & $9.2(5.2,14.5)$ & 0.06 \\
\hline & $\mathrm{SpO}_{2}$ & INVOS & FORE-SIGHT & \\
\hline INVOS/FORE-SIGHT $(n=4)$ & $(\% /$ min $)$ & $(\% / \min )$ & $(\% / \min )$ & $P$ value \\
\hline Resaturation \#1 & $12.9(10.1,18.8)$ & $10.6(8.1,19.3)$ & $9.5(6.5,14.4)$ & 0.10 \\
\hline Resaturation \#2 & $11.7(10.4,13.1)$ & $9.6(5.8,12.5)$ & $6.6(4.4,12.0)$ & 0.20 \\
\hline
\end{tabular}

Data are unavailable for one INVOX/EQUANOX subject. ${ }^{a}$ Indicates statistical significance between devices (two-tailed Wilcoxon Signed-Rank test for paired samples)

improve outcomes and see different results from published trials if the same device is not used. We make no judgment about which device is "correct," simply that they are different from one another.

Device design may contribute to the differences in reporting $\mathrm{rSO}_{2}$ during changes in cerebral oxygen saturation. Manufacturers make unique assumptions of arterial versus venous contribution of the tissue under the sensor: 25/75 for INVOS, and 30/70 for EQUANOX and FORE-SIGHT. Each device uses a distinct proprietary algorithm. INVOS 5100C has a 2-wavelength LED light source in the sensor, compared with a 3-wavelength LED in EQUANOX 7600 and a 4-wavelength laser in FORE-SIGHT. The devices also sample different tissue depths due to individual sensor/detector spacing.

Our findings corroborate those published from previous device comparisons in both healthy subjects and surgical patients, with some exceptions. Fellahi and colleagues found a greater percent maximum difference from baseline with INVOS compared with EQUANOX in healthy subjects during leg vascular occlusion tests [16]. In 42 off-pump coronary artery bypass surgery patients, Moerman and colleagues reported a greater area under the curve for INVOS during desaturation compared to FORE-SIGHT (-68\%/s vs $-39 \% / \mathrm{s})$ and only a weak correlation in $\mathrm{rSO}_{2}$ values between the two devices $(r=0.31)$ [17]. In contrast, Closhen and colleagues found similar changes in $\mathrm{rSO}_{2}$ between INVOS and FORE-SIGHT in 35 patients moved into the beachchair position for arthroscopic shoulder surgery, although the absolute change in $\mathrm{rSO}_{2}$ for both devices during repositioning was minimal (10\%) [18]. The authors reported a stronger correlation in $\mathrm{rSO}_{2}$ values between the two devices $(r=0.68)$ than Moerman and colleagues. Both Moerman and Fellahi reported a wider range of values from INVOS compared with FORESIGHT and EQUANOX, respectively [16, 17].

Fellahi and colleagues also found significantly greater rates of $\mathrm{rSO}_{2}$ change during desaturation $(3.65 \% / \mathrm{min}$ vs $2.36 \% / \mathrm{min})$ and resaturation (30.4 vs $16.8 \% / \mathrm{min})$ for INVOS compared with EQUANOX, similar to our own findings [16]. But Hyttel-Sorensen and colleagues reported a steeper desaturation slope with EQUANOX during arm vascular occlusion in 10 healthy volunteers compared with INVOS and FORE-SIGHT [19].

Few studies compared how and when different devices detect clinically relevant desaturation events. Pisano et al., compared devices in cardiac surgery and reported that INVOS detected 20 significant cerebral desaturation events in four of ten patients, compared with three events in one patient for EQUANOX (the same patient in which INVOS detected five events) [20]. Unlike our study, two sensors per device were placed bilaterally, with one sensor pair placed above the other on the forehead. We cannot compare these results to ours since Pisano et al., recorded $\mathrm{rSO}_{2}$ from only one device at a time due to interference, and as such would not have detected the same desaturation events. In Closhen's beach-chair study, INVOS reported one event of $\mathrm{rSO}_{2}$ less than $50 \%$ during positioning that was not reported by FORE-SIGHT [18]. In a multicenter, randomized, controlled study of high-risk cardiac surgery patients where all three devices were used, Deschamps et al., found that INVOS detected on average 2.3 desaturation events per patient, compared with 1.7 for EQUANOX, and 1.5 for FORE-SIGHT, although only one device was

Table 4 Detection of critical $\mathrm{rSO}_{2}$ thresholds during the first desaturation cycle $(n, \%)$

\begin{tabular}{|c|c|c|c|c|c|c|}
\hline & $\begin{array}{l}\text { INVOS: YES } \\
\text { EQUANOX: NO } \\
n=6\end{array}$ & $\begin{array}{l}\text { INVOS: NO } \\
\text { EQUANOX: YES } \\
n=6\end{array}$ & $P$ value & $\begin{array}{l}\text { INVOS: YES } \\
\text { FORE-SIGHT: NO } \\
n=4\end{array}$ & $\begin{array}{l}\text { INVOS: NO } \\
\text { FORE-SIGHT: YES } \\
n=4\end{array}$ & $P$ value \\
\hline $20 \%$ relative $\mathrm{rSO}_{2}$ decrease from baseline met & $5(83 \%)$ & 0 & 0.06 & $2(50 \%)$ & 0 & 0.50 \\
\hline $50 \%$ absolute $\mathrm{rSO}_{2}$ threshold met & $4(67 \%)$ & 0 & 0.06 & $3(75 \%)$ & 0 & 0.25 \\
\hline
\end{tabular}

Data were not statistically significant (exact binomial test) 
used per patient so there were no direct comparisons of detecting the same event [2].

We point out several important limitations in our study for consideration. We were not able to place the sensors $2 \mathrm{~cm}$ apart on all subjects, and we did not investigate whether there was interference between the devices at close distances. We did not calculate a sample size a priori based on a predetermined difference between devices in either the magnitude or rate of $\mathrm{rSO}_{2}$ change. In cases where we observed no significant difference between devices, we are unsure if there truly was no difference, or if our study lacked sufficient power. We tend to believe the latter, particularly with the 4subject INVOS/FORE-SIGHT cohort.

Also, we suggest interpreting differences in $\mathrm{rSO}_{2}$ rates of change during desaturation in our study with caution; the anesthesiologist titrated $\mathrm{FiO}_{2}$ gradually as the subject tolerated it, which may introduce between-subject variation. Baseline $\mathrm{rSO}_{2}$ reported by INVOS was notably lower in some subjects than EQUANOX and FORESIGHT, potentially influencing whether a device reached the $50 \%$ absolute $\mathrm{rSO}_{2}$ threshold during desaturation. Also, unlike two of the papers cited here, we used each device unilaterally $[17,20]$. We concede that one device could have reached a threshold earlier than (or in the absence of) another due to hemispheric differences. Finally, we did not directly sample arterial and venous blood for calculating a weighted saturation as a reference to compare with $\mathrm{rSO}_{2}$ values. As such, we cannot comment on which device best represented cerebral oxygen status. But despite the small nature of our pilot study, the hypoxia protocol reported here is repeatable and widely used for validating regional oximeters for agency approval. We generated intriguing preliminary results to inform future hypotheses and hopefully generate interest in larger, more comprehensive device comparisons.

With these preliminary results, one might consider whether clinical evidence generated in one manufacturer's regional oximetry device can be broadly applied to similar devices from other manufacturers. The three devices tested in our study reached critical interventional $\mathrm{rSO}_{2}$ thresholds inconsistently in the same subject. Although the differences between devices in detecting critical $\mathrm{rSO}_{2}$ thresholds were not statistically significant, we argue that the results may have clinical significance. A larger study with adequate power may clarify our findings.

\section{Conclusions}

To the best of our knowledge, our report is the first to show differences between INVOS and both FORESIGHT and EQUANOX in detecting the same desaturation event when subjects are monitored simultaneously. With this knowledge, one should take care in broadly applying evidence of improved patient outcomes to all devices when a single manufacturer's device is used in a study. Larger studies in clinical settings are required to investigate the clinical impact of this finding.

\section{Additional files}

\author{
Additional file 1: Raw data subject \#2234. (XLSX 107 kb) \\ Additional file 2: Raw data subject \#1228. (XLSX 123 kb) \\ Additional file 3: Raw data subject \#1654. (XLSX 174 kb) \\ Additional file 4: Raw data subject \#1657. (XLSX 122 kb) \\ Additional file 5: Raw data subject \#1667. (XLSX 139 kb) \\ Additional file 6: Raw data subject \#1791. (XLSX 116 kb) \\ Additional file 7: Raw data subject \#1806. (XLSX 128 kb) \\ Additional file 8: Raw data subject \#1824. (XLSX 118 kb) \\ Additional file 9: Raw data subject \#2142. (XLSX 124 kb) \\ Additional file 10: Raw data subject \#2174. (XLSX $112 \mathrm{~kb}$ ) \\ Additional file 11: Tabulated data from all subjects. (XLSX $62 \mathrm{~kb}$ )
}

\section{Abbreviations}

$\mathrm{FeO}_{2}$ : Fraction of exhaled oxygen; $\mathrm{FiO}_{2}$ : Fraction of inhaled oxygen; $\mathrm{rSO}_{2}$ : Cerebral oxygen saturation; $\mathrm{SpO}_{2}$ : Peripheral capillary oxygen saturation

\section{Acknowledgements}

The authors recognize Monika Deshpande, MSc, for helping to coordinate the study, recruit subjects, obtain informed consent, and collect data; Yu-Jung Pinto, MSc, for providing all data in synchronized formats; Zengri Wang, PhD, for review of the statistical analyses; and Michael Mestek, PhD, for critical review of the manuscript.

\section{Funding}

Medtronic funded the study. Medtronic employees designed the study; collected, analyzed, and interpreted the data; and wrote the manuscript.

\section{Availability of data and materials}

Datasets have been provided as Additional files 1, 2, 3, 4, 5, 6, 7, 8, 9, 10 and 11 as part of the manuscript submission.

\section{Authors' contributions}

AMN and UB developed the protocol, coordinated the study, recruited subjects, and gathered informed consent; collected and interpreted data; and helped to critically revise the manuscript. KLT helped interpret and analyze the data, performed statistical analyses, wrote the first draft and revised subsequent drafts of the manuscript. All authors have read and approved of the final manuscript.

\section{Authors' information}

All authors are employees of the Patient Monitoring \& Recovery Medical Affairs Department at Medtronic (Boulder, Colorado, USA). KLT is a Medical Affairs Program Manager and holds a PhD in cellular, molecular, and microbial biology. AMN is a Clinical Program Manager holds an MD with a specialty in general medicine. UB is the Director of Clinical Science and a Registered Respiratory Therapist with extensive clinical experience in critical care, and human and animal research.

\section{Competing interests}

The authors declare that they are employees of Medtronic and hold Medtronic stock.

Consent for publication

Not applicable.

Ethics approval and consent to participate

The Western Institutional Review Board (Puyallup, WA) approved an overarching protocol for a study of pulse oximetry devices, under which this 
was a substudy (protocol \#070808; Non-invasive Controlled Acute Hypoxia Studies). We obtained informed consent from all subjects prior to participating in the study.

Received: 24 August 2016 Accepted: 27 December 2016

Published online: 13 January 2017

\section{References}

1. Zacharias DG, Lilly K, Shaw CL, Pirundini P, Rizzo RJ, Body SC, Longford NT. Survey of the clinical assessment and utility of near-infrared cerebral oximetry in cardiac surgery. J Cardiothorac Vasc Anesth. 2014;28(2):308-16.

2. Deschamps A, Hall R, Grocott H, Mazer CD, Choi PT, Turgeon A, de Medici E, Bussieres JS, Hudson C, Syed S, et al. Cerebral Oximetry Monitoring to Maintain Normal Cerebral Oxygen Saturation during High-risk Cardiac Surgery: A Randomized Controlled Feasibility Trial. Anesthesiology. 2016; 124(4):826-36

3. Pant S, Bokor DJ, Low AK. Cerebral oxygenation using near-infrared spectroscopy in the beach-chair position during shoulder arthroscopy under general anesthesia. Arthroscopy. 2014;30(11):1520-7.

4. Casati A, Fanelli G, Pietropaoli P, Proietti R, Tufano R, Danelli G, Fierro G, De Cosmo G, Servillo G. Continuous monitoring of cerebral oxygen saturation in elderly patients undergoing major abdominal surgery minimizes brain exposure to potential hypoxia. Anesth Analg. 2005;101(3):740-7. table of contents.

5. Salazar F, Donate M, Boget T, Bogdanovich A, Basora M, Torres F, Gracia I, Fabregas N. Relationship between intraoperative regional cerebral oxygen saturation trends and cognitive decline after total knee replacement: a posthoc analysis. BMC Anesthesiol. 2014;14:58.

6. Denault A, Deschamps A, Murkin JM. A proposed algorithm for the intraoperative use of cerebral near-infrared spectroscopy. Semin Cardiothorac Vasc Anesth. 2007;11(4):274-81.

7. Denault A, Lamarche Y, Rochon A, Cogan J, Liszkowski M, Lebon JS, Ayoub C, Taillefer J, Blain R, Viens C, et al. Innovative approaches in the perioperative care of the cardiac surgical patient in the operating room and intensive care unit. Can J Cardiol. 2014;30(12 Suppl):S459-77.

8. Kara I, Erkin A, Sacli H, Demirtas M, Percin B, Diler MS, Kirali K. The Effects of Near-Infrared Spectroscopy on the Neurocognitive Functions in the Patients Undergoing Coronary Artery Bypass Grafting with Asymptomatic Carotid Artery Disease: A Randomized Prospective Study. Ann Thorac Cardiovasc Surg. 2015;21(6):544-50.

9. Vretzakis G, Georgopoulou S, Stamoulis K, Tassoudis V, Mikroulis D, Giannoukas A, Tsilimingas N, Karanikolas M. Monitoring of brain oxygen saturation (INVOS) in a protocol to direct blood transfusions during cardiac surgery: a prospective randomized clinical trial. J Cardiothorac Surg. 2013:8:145.

10. Colak Z, Borojevic M, Bogovic A, Ivancan V, Biocina B, Majeric-Kogler V. Influence of intraoperative cerebral oximetry monitoring on neurocognitive function after coronary artery bypass surgery: a randomized, prospective study. Eur J Cardiothorac Surg. 2015:47(3):447-54.

11. Mohandas BS, Jagadeesh AM, Vikram SB. Impact of monitoring cerebral oxygen saturation on the outcome of patients undergoing open heart surgery. Ann Card Anaesth. 2013;16(2):102-6.

12. Murkin JM, Adams SJ, Novick RJ, Quantz M, Bainbridge D, Iglesias I, Cleland A, Schaefer B, Irwin B, Fox S. Monitoring brain oxygen saturation during coronary bypass surgery: a randomized, prospective study. Anesth Analg. 2007:104(1):51-8.

13. Murkin JM, Adams SJ, Pardy E, Quantz M, McKenzie FN, Guo L. Monitoring brain oxygen saturation during coronary bypass surgery improves outcomes in diabetic patients: a post hoc analysis. Heart Surg Forum. 2011; 14(1):E1-6.

14. Ghosh A, Elwell C, Smith M. Review article: cerebral near-infrared spectroscopy in adults: a work in progress. Anesth Analg. 2012;115(6):1373-83.

15. Steppan J, Hogue Jr CW. Cerebral and tissue oximetry. Best Pract Res Clin Anaesthesiol. 2014;28(4):429-39.

16. Fellahi JL, Butin G, Fischer MO, Zamparini G, Gerard JL, Hanouz JL. Dynamic evaluation of near-infrared peripheral oximetry in healthy volunteers: a comparison between INVOS and EQUANOX. J Crit Care. 2013;28(5):881.e881-886

17. Moerman A, Vandenplas G, Bove T, Wouters PF, De Hert SG. Relation between mixed venous oxygen saturation and cerebral oxygen saturation measured by absolute and relative near-infrared spectroscopy during offpump coronary artery bypass grafting. Br J Anaesth. 2013;110(2):258-65.
18. Closhen D, Berres M, Werner C, Engelhard K, Schramm P. Influence of beach chair position on cerebral oxygen saturation: a comparison of INVOS and FORE-SIGHT cerebral oximeter. J Neurosurg Anesthesiol. 2013;25(4):414-9.

19. Hyttel-Sorensen S, Hessel TW, Greisen G. Peripheral tissue oximetry: comparing three commercial near-infrared spectroscopy oximeters on the forearm. J Clin Monit Comput. 2014;28(2):149-55.

20. Pisano A, Galdieri N, lovino TP, Angelone M, Corcione A. Direct comparison between cerebral oximetry by INVOS(TM) and EQUANOX(TM) during cardiac surgery: a pilot study. Heart Lung Vessel. 2014;6(3):197-203.

\section{Submit your next manuscript to BioMed Central and we will help you at every step:}

- We accept pre-submission inquiries

- Our selector tool helps you to find the most relevant journal

- We provide round the clock customer support

- Convenient online submission

- Thorough peer review

- Inclusion in PubMed and all major indexing services

- Maximum visibility for your research

Submit your manuscript at www.biomedcentral.com/submit
Biomed Central 